\title{
Colored lenses suppress blue light- emitting diode light-induced damage in photoreceptor-derived cells
}

Kaho Hiromoto

Yoshiki Kuse

Kazuhiro Tsuruma

Nobuyuki Tadokoro

Nobuyuki Kaneko

Masamitsu Shimazawa

Hideaki Hara 


\title{
Colored lenses suppress blue light-emitting diode light-induced damage in photoreceptor-derived cells
}

\author{
Kaho Hiromoto, ${ }^{a}$ Yoshiki Kuse,${ }^{a}$ Kazuhiro Tsuruma, ${ }^{a}$ Nobuyuki Tadokoro, ${ }^{b}$ Nobuyuki Kaneko, ${ }^{b}$ \\ Masamitsu Shimazawa, ${ }^{a}$ and Hideaki Haraa,* \\ ${ }^{a}$ Gifu Pharmaceutical University, Molecular Pharmacology, Department of Biofunctional Evaluation, 1-25-4 Daigakunishi, Gifu 501-1196, Japan \\ ${ }^{b}$ HOYA Corporation, VC Section, CS Support Division, Japan Headquarters, 4-10-2 Nakano, Nakanoku, Tokyo 164-8545, Japan
}

\begin{abstract}
Blue light-emitting diodes (LEDs) in liquid crystal displays emit high levels of blue light, exposure to which is harmful to the retina. Here, we investigated the protective effects of colored lenses in blue LED lightinduced damage to $661 \mathrm{~W}$ photoreceptor-derived cells. We used eight kinds of colored lenses and one lens that reflects blue light. Moreover, we evaluated the relationship between the protective effects of the lens and the transmittance of lens at $464 \mathrm{~nm}$. Lenses of six colors, except for the SY, PN, and reflective coating lenses, strongly decreased the reduction in cell damage induced by blue LED light exposure. The deep yellow lens showed the most protective effect from all the lenses, but the reflective coating lens and pink lens did not show any effects on photoreceptor-derived cell damage. Moreover, these results were correlated with the lens transmittance of blue LED light $(464 \mathrm{~nm})$. These results suggest that lenses of various colors, especially deep yellow lenses, may protect retinal photoreceptor cells from blue LED light in proportion to the transmittance for the wavelength of blue LED and the suppression of reactive oxygen species production and cell damage. ( ) The Authors. Published by SPIE under a Creative Commons Attribution 3.0 Unported License. Distribution or reproduction of this work in whole or in part requires full attribution of the original publication, including its DOI. [DOI: 10.1117/1.JBO.21.3.035004]
\end{abstract}

Keywords: cells; diodes; lenses; correlation.

Paper 150714RR received Oct. 26, 2015; accepted for publication Mar. 2, 2016; published online Mar. 23, 2016.

\section{Introduction}

Blue light-emitting diodes (LEDs) are semiconductor devices that are commonly used as light sources in LED-backlit liquid crystal displays of various electronic appliances such as smartphones, computer screens, and LED lamps. As compared with a normal lamp, the LED lamp has several advantages, such as less heat, longer life, and good energy efficiency. Blue LEDs also display several other properties apart from being a light source. Blue light has been reported to be lethal to insects. ${ }^{1}$ Additionally, blue LED light is shown to display a therapeutic effect in seasonal affective disorder. ${ }^{2}$ However, blue LED emits only short-wavelength high-energy visible light and long-time video display terminal works expose human eyes to blue LED light. Further, its night-time exposure can suppress the secretion of melatonin, resulting in sleep disorders. ${ }^{3-5}$ Moreover, exposure to blue LED light leads to increased production of reactive oxygen species (ROS) ${ }^{6}$ Oxidative stress induced by ROS is known to trigger photoreceptor cell ${ }^{7}$ and retinal pigment epithelium (RPE) cell death. ${ }^{8}$ Oxidative stressinduced RPE cell death may become a risk factor for age-related macular degeneration (AMD), which is the main cause of blindness in industrialized nations. ${ }^{9,10}$ Therefore, overexposure to blue light may be a risk for the progression of AMD. ${ }^{8,9}$ While wet AMD can be treated by several antiangiogenic drugs such as Ranibizumab, ${ }^{11}$ there is no effective treatment yet for dry AMD.

One of the easiest ways to reduce the risks of acquiring blue light exposure-mediated sleep disorder and $\mathrm{AMD}^{5,12}$ is to wear blue light-blocking lenses. A blue light-cutting lens is a yellow lens, which absorbs almost all blue light, or a colorless lens,

*Address all correspondence to: Hideaki Hara, E-mail: hidehara@ gifu-pu.ac.jp whose surface is processed to reflect blue light. In this study, the protective effect of light colored lenses on a blue LED light-induced murine photoreceptor-derived cell damage model was investigated by evaluating cell viability, the rate of cell death, and ROS production. Some colored lenses had a protective effect in this model. Notably, our findings showed that the transmittance of blue light is in large correlation with the protective effect of colored lenses in a blue LED light-induced cell damage model. This result strongly suggested that the consideration of an amount of blue light was important in the protection of eyes from light-related eye diseases such as AMD.

\section{Materials and Methods}

\subsection{Cell Culture}

The murine photoreceptor-derived cell line $(661 \mathrm{~W})$ was kindly gifted by Dr. Muayyad R. Al-Ubaidi (Department of Cell Biology, University of Oklahoma Health Sciences Center, Oklahoma City, Oklahoma).

The cells were cultured in Dulbecco's modified Eagle medium (DMEM; Wako, Osaka, Japan) containing 10\% fetal bovine serum (FBS; Sigma-Aldrich, St. Louis, Missouri), $100 \mathrm{U} / \mathrm{mL}$ penicillin (Meiji Seika Kaisha Ltd., Tokyo, Japan), and $100 \mu \mathrm{g} / \mathrm{mL}$ streptomycin (Meiji Seika) under 5\% $\mathrm{CO}_{2}$ atmosphere at $37^{\circ} \mathrm{C}$. The cells were passaged every 2 to 3 days by trypsinization.

\subsection{Photoreceptor-Derived Cell Damage Induced by Blue Light-Emitting Diode}

The $661 \mathrm{~W}$ cells were seeded into 96 -well plates at a density of $3 \times 10^{3}$ cells per well and incubated for $24 \mathrm{~h}$ under $5 \% \mathrm{CO}_{2}$ atmosphere at $37^{\circ} \mathrm{C}$. Following this, the cell culture medium 
was replaced by DMEM containing $1 \%$ FBS, and the plates were exposed to 350 to 800 lux blue LED light for $24 \mathrm{~h}$. The wavelength of blue LED light was $464 \mathrm{~nm} .{ }^{13}$ Control groups were shaded by aluminum foil and lens groups were placed on lenses, while exposing experimental groups to blue LED light.

\subsection{Colored Lenses}

RETINEX lenses, blue light-reflecting lenses, and Y50, the cutting filter below $500 \mathrm{~nm}$ (HOYA, Tokyo, Japan), were used for all experiments. Evaluated colors were Y50 filter, YE, SYD, OO, OG, GN, SY, PN, and blue light-reflecting lenses (Fig. 1).

\subsection{Cell Death Analysis by Hoechst 33342 and Propidium lodide Staining}

The $661 \mathrm{~W}$ cells were seeded in 96-well plates at a density of $3 \times$ $10^{3}$ cells per well and incubated for $24 \mathrm{~h}$ in $5 \% \mathrm{CO}_{2}$ at $37^{\circ} \mathrm{C}$. The cell culture medium was replaced by DMEM containing $1 \%$ FBS, and the plates were exposed to 350 to 800 lux blue LED light for $24 \mathrm{~h}$. Following LED exposure, the plates were incubated for $12 \mathrm{~h}$ in $5 \% \mathrm{CO}_{2}$ at $37^{\circ} \mathrm{C}$, and the cells were stained for 15 min with Hoechst 33342 (Molecular Probes, Eugene,
Oregon) and propidium iodide (PI; Molecular Probes). Hoechst 33342 and PI were added in culture wells at final concentrations of 8.1 and $1.5 \mu \mathrm{M}$, respectively. The stained cells were observed and images were captured by Olympus IX70 inverted epifluorescence microscope (Olympus, Tokyo, Japan).

\subsection{Cell Viability Assay}

Cell viability was assayed using CCK-8 (Dojin Kagaku, Kumamoto, Japan). The $661 \mathrm{~W}$ cells were seeded into 96well plates at a density of $3 \times 10^{3}$ cells per well and incubated for $24 \mathrm{~h}$ in $5 \% \mathrm{CO}_{2}$ at $37^{\circ} \mathrm{C}$. Following this, the cell culture medium was replaced by DMEM containing $1 \%$ FBS, and the plates were exposed to 350 to 800 lux blue LED light for $24 \mathrm{~h}$. Subsequently, CCK- 8 reagents $(10 \mu \mathrm{L} /$ well) were added in each well and incubated for 0 to $2 \mathrm{~h}$, after which the optical density at $450 \mathrm{~nm}$ was measured with a microplate reader (Varioskan Flash 2.4; Thermo Fisher Scientific, Waltham, Massachusetts).

\subsection{Measurement of Reactive Oxygen Species Production}

The $661 \mathrm{~W}$ cells were seeded into $96-$ well plates at a density of $3 \times 10^{3}$ cells per well and incubated for $24 \mathrm{~h}$ in $5 \% \mathrm{CO}_{2}$

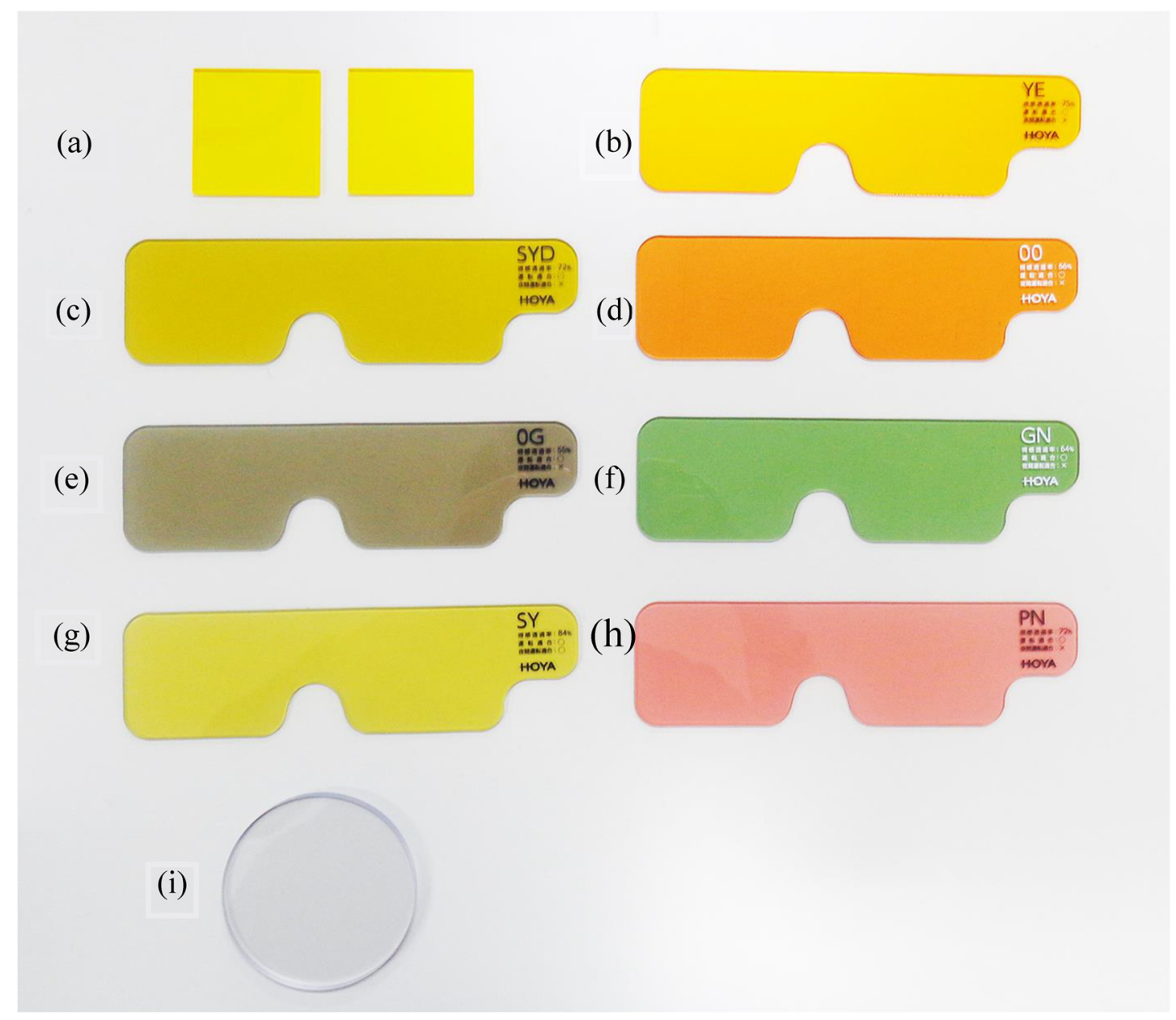

Fig. 1 Various colored lenses used in this study: (a) Y50 filter, (b) YE lens, (c) SYD lens, (d) OO lens, (e) OG lens, (f) GN lens, (g) SY lens, (h) PN lens, and (i) blue light antireflective coating lens. 
at $37^{\circ} \mathrm{C}$. Following this, the culture medium was replaced by DMEM containing $1 \%$ FBS, and the plates were exposed to 350 to 800 lux blue LED light for $24 \mathrm{~h}$. ROS were then measured by 5-(and-6)-chloromethyl-2',7'-dichlorodihydrofluorescein diacetate acetyl ester (CM- $\mathrm{H}_{2} \mathrm{DCFDA}$; Eugene, Oregon). CM- $\mathrm{H}_{2}$ DCFDA $(10 \mu \mathrm{M})$ was added to the wells and incubated for 0 to $1 \mathrm{~h}$ in $5 \% \mathrm{CO}_{2}$ at $37^{\circ} \mathrm{C}$. Fluorescence was measured by a microplate reader at $485 / 535 \mathrm{~nm}$. The number of live cells was counted by Hoechst and PI staining.

\subsection{Statistical Analysis}

Data are presented as the mean \pm S.E.M. Statistical comparisons were made by one-way ANOVA followed by Tukey's test. $p<0.05$ was considered statistically significant. Correlation value was calculated by Pearson product-moment correlation coefficient.

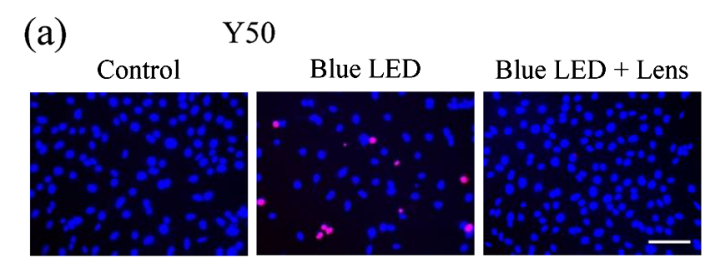

(c)
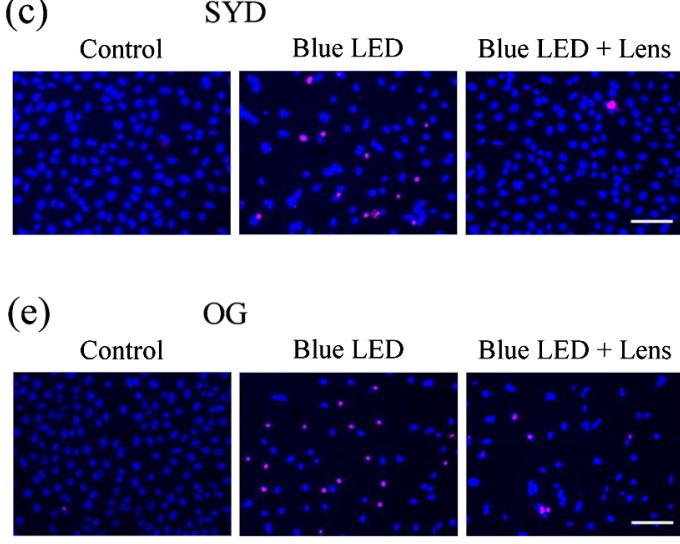

OG
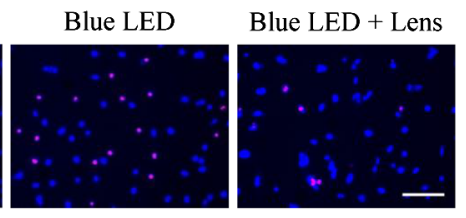

(g) SY
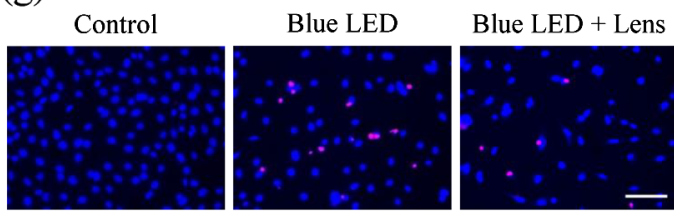

(i)

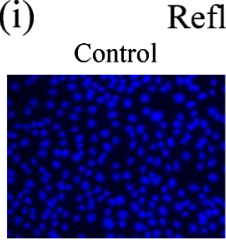

Reflective coating

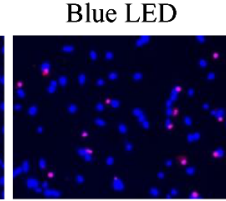

Blue LED + Lens

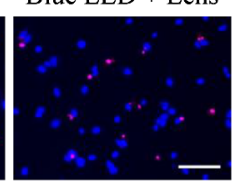

(h)

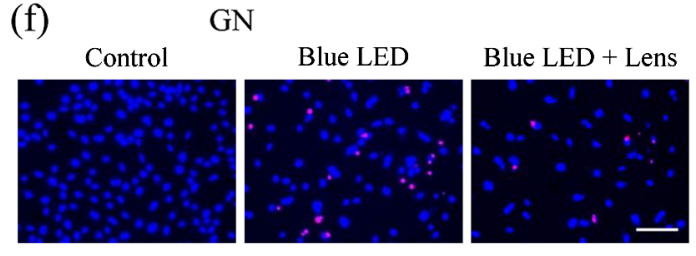

GN
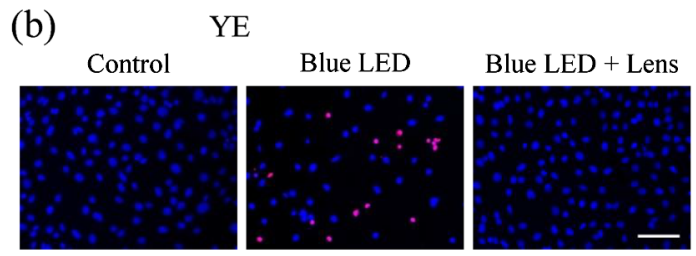

(d)

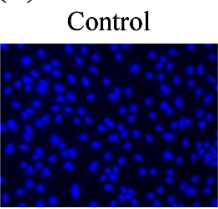

$\mathrm{OO}$
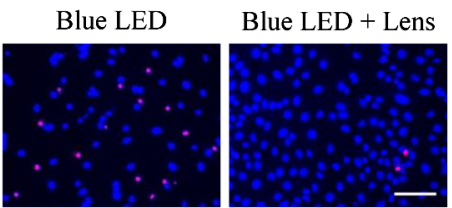

\section{Results}

\subsection{Rate of Cell Death Was Decreased in Several}

The rate of cell death was calculated based on Hoechst 33342 positive and PI positive cells, and typical fluorescence microsche shown in Fig. 2 . The rate of cell death in the norate of cell death in the Y50 filter, YE, SYD, OO, OG, GN, SY, PN lens groups was significantly decreased in comparison the reflective coating lens group did not show any decrease as en no-lens group [Fig. 3(i)]

\subsection{Several Lens Groups Improved Cell Viability}

Cell viability was measured by CCK-8 and was observed to decrease upon blue LED light exposure. In comparison

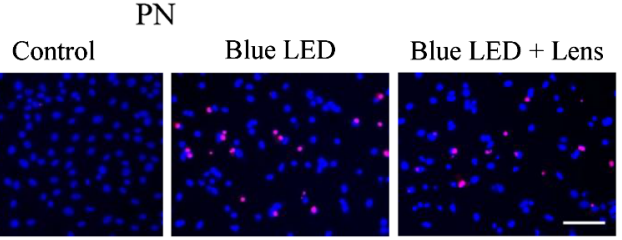

Fig. 2 Effect of colored lenses on blue LED light-induced damage in $661 \mathrm{~W}$ cells. Fluorescence microscopy images after staining with Hoechst 33342 (blue) and PI (red). Live cells were stained with Hoechst 33342, and dead cells were stained with Hoechst 33342 and PI. (a) Y50 filter, (b) YE, (c) SYD, (d) OO, (e) OG, (f) GN, (g) SY, and (h) PN lenses decreased the number of dead cells. (i) Reflective coating lenses did not decrease the number of dead cells. Scale bar $=100 \mu \mathrm{m}$. 
(a)

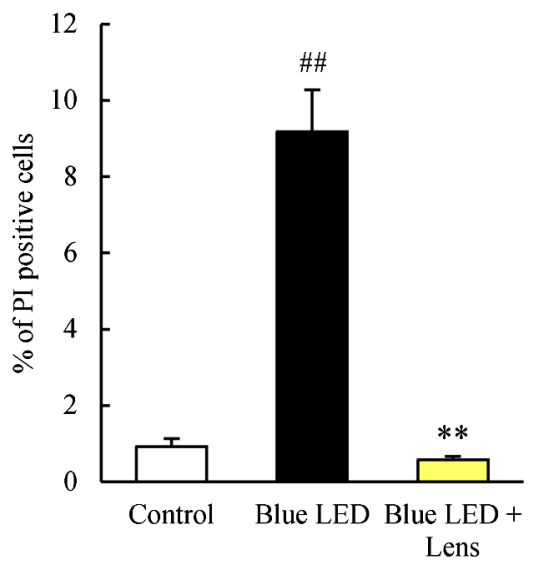

(d)

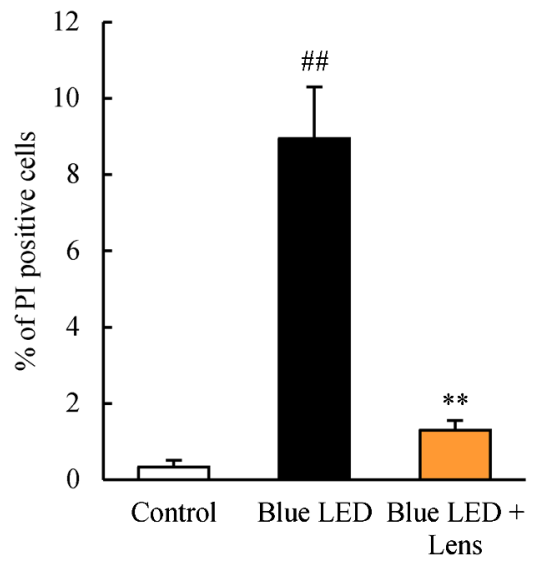

$(\mathrm{g})$

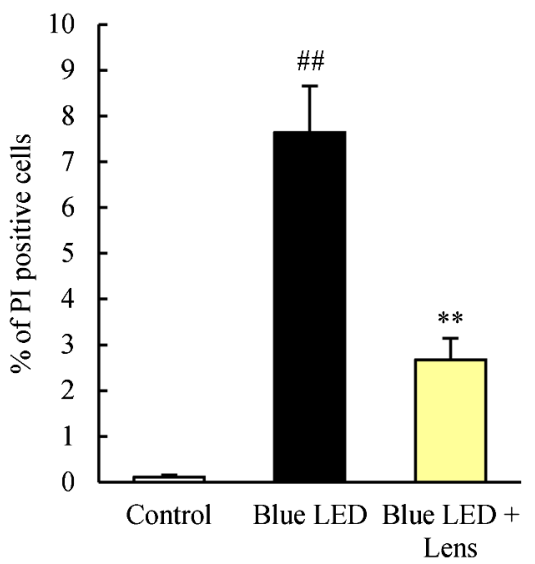

(b)

YE

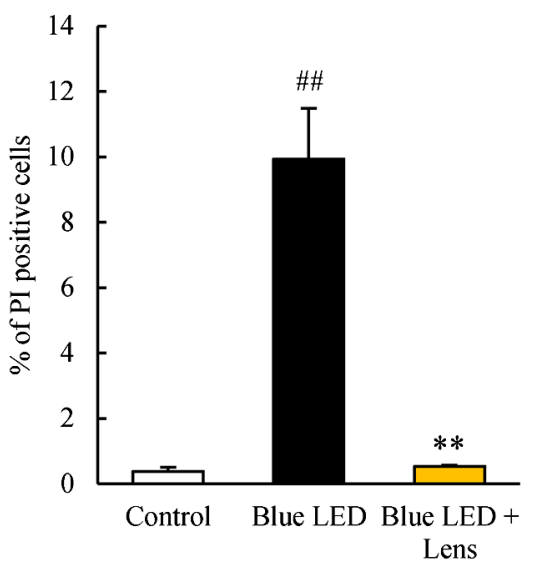

(e)

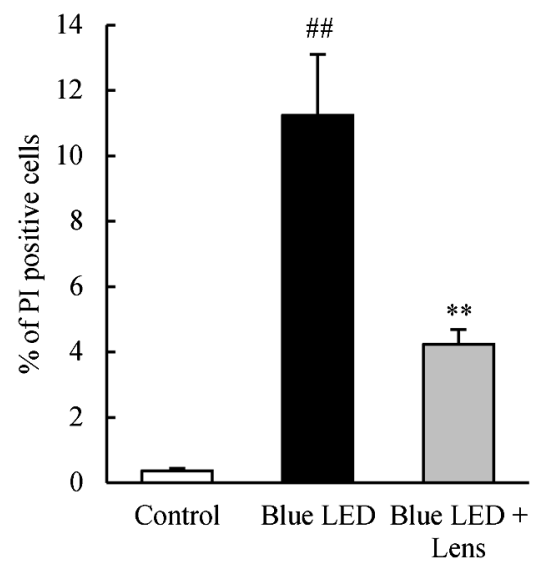

(h)

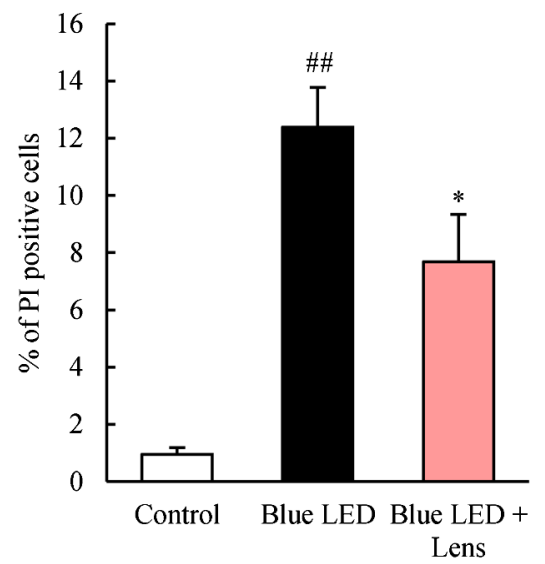

(c)

SYD

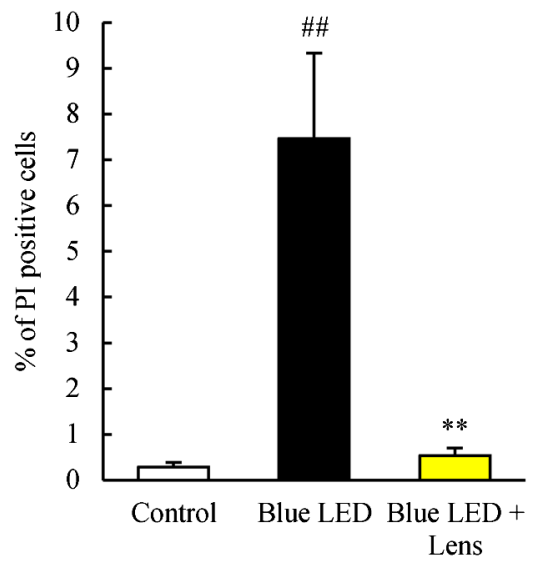

(f)

GN

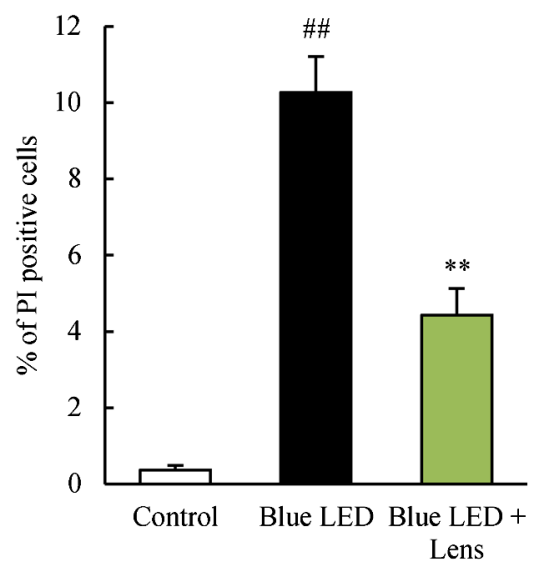

(i)

Reflective coating

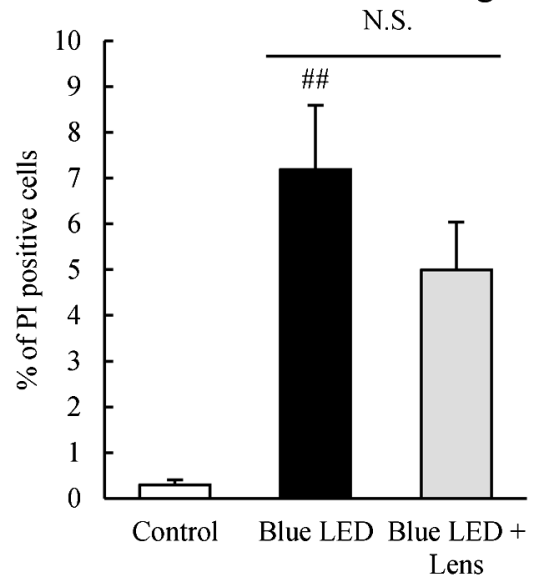

Fig. 3 Effect of colored lenses on the rate of cell death upon blue LED light-induced damage in 661W cells. The total number of Hoechst 33342 and PI positive cells was counted, and the rate of cell death was calculated as the percentage of PI positive cells to the number of total cells. Rate of cell death increased upon blue LED light exposure. (a) Y50 filter, (b) YE, (c) SYD, (d) OO, (e) OG, (f) GN, (g) SY, and (h) PN lenses decreased the rate of cell death in comparison with the no-lens group. (i) Reflective coating lenses did not decrease the rate of cell death compared with the no-lens group. Data are expressed as mean $\pm \operatorname{SEM}(n=6)$. ${ }^{\# \#} p<0.01$ versus control; ${ }^{*} p<0.05$ versus blue LED light exposure; and ${ }^{* *} p<0.01$ versus blue LED light exposure (one-way ANOVA followed by Tukey's test). 


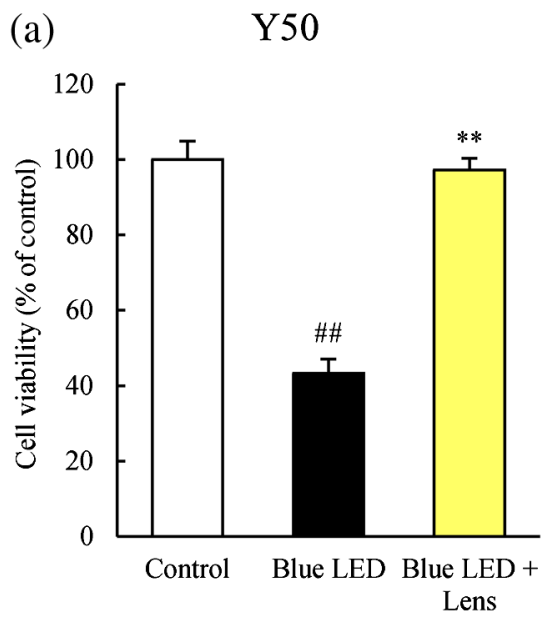

(d)

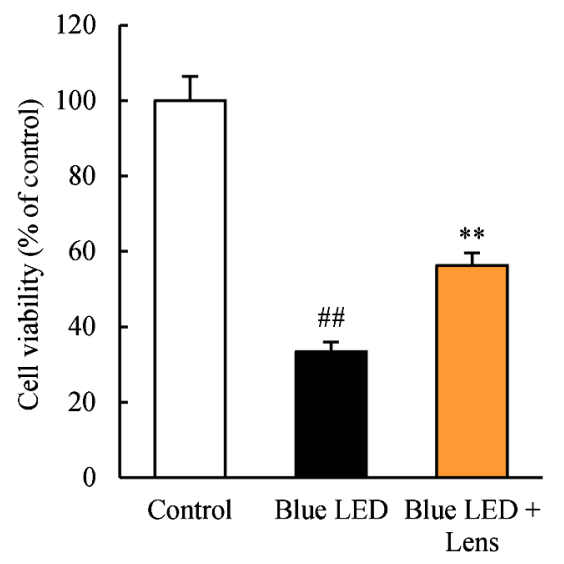

(g)

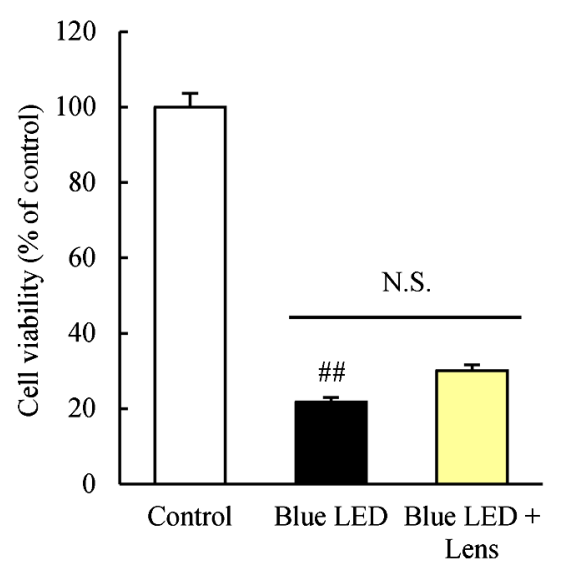

(b)

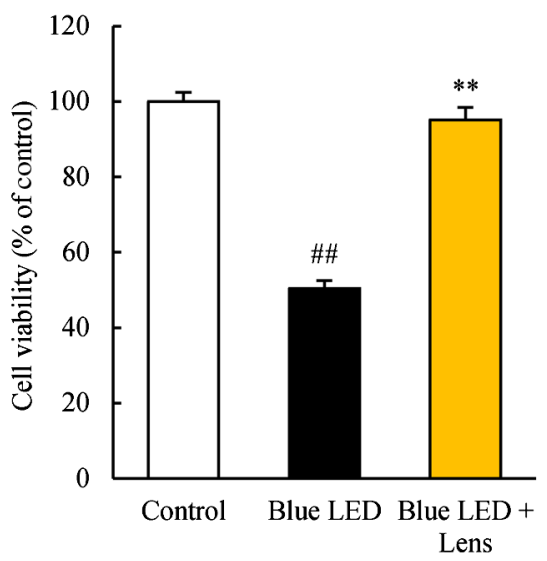

(e)

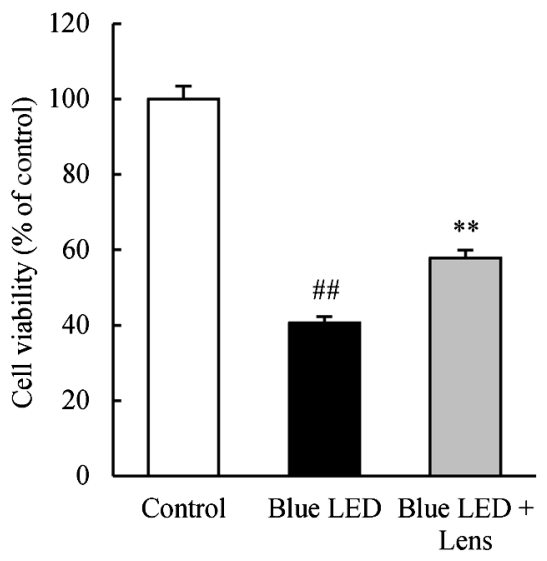

(h)

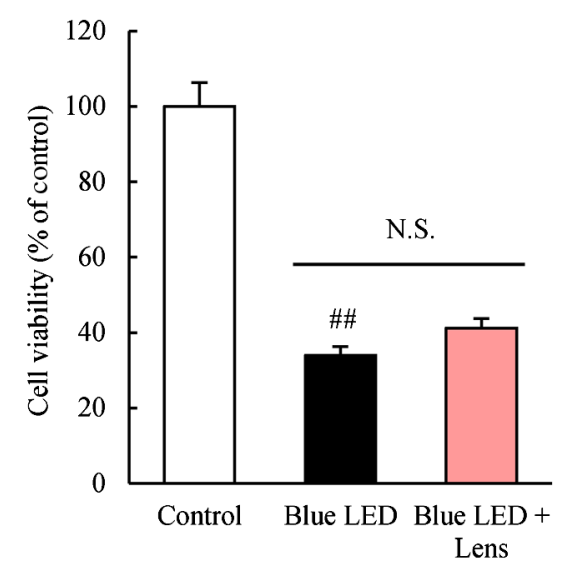

(c)

SYD

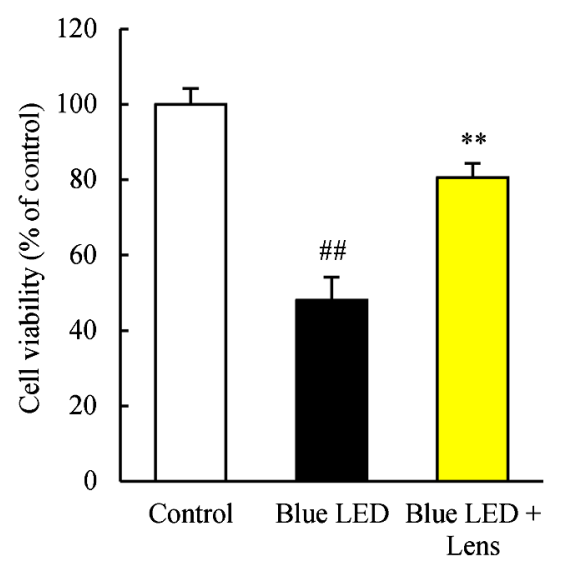

(f)

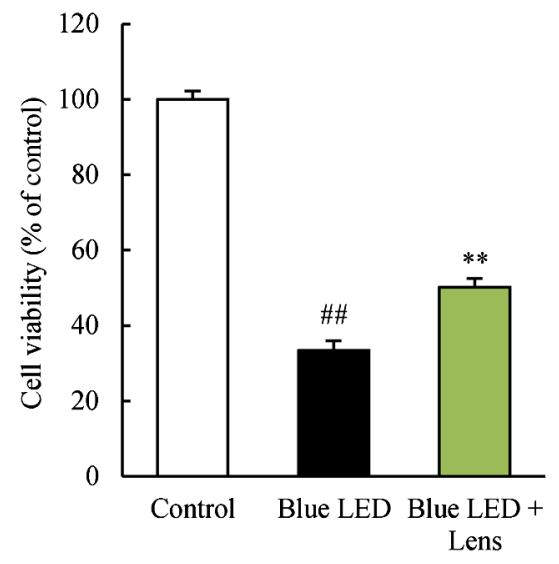

(i) Reflective coating

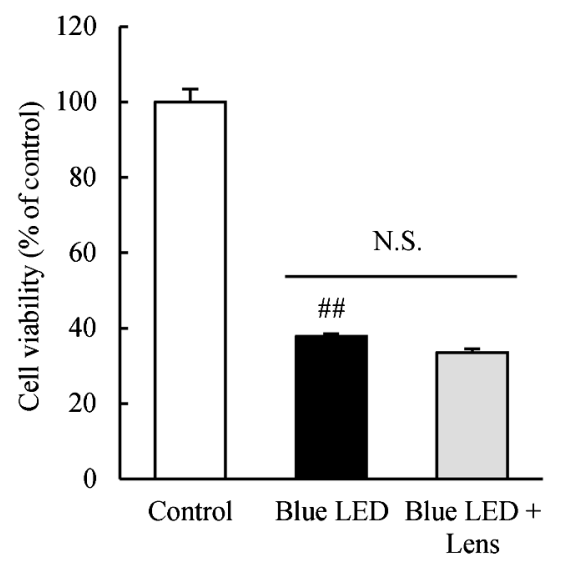

Fig. 4 Effect of colored lenses on cell viability upon blue LED light-induced damage in $661 \mathrm{~W}$ cells. Cell viability was assayed by CCK-8 and was reduced upon blue LED light exposure. (a) Y50 filter, (b) YE, (c) SYD, (d) OO, (e) OG, and (f) GN lenses significantly improved cell viability compared with the no-lens group. (g) SY, (h) PN, and (i) reflective coating lenses did not improve cell viability compared with the nolens group. Data are expressed as mean $\pm \operatorname{SEM}(n=6)$. ${ }^{\# \#} p<0.01$ versus control; and ${ }^{* *} p<0.01$ versus blue LED light exposure (one-way ANOVA followed by Tukey's test).

with the control group, the cell viability in the no-lens group decreased by $30 \%$ to $50 \%$ (Fig. 4). Further, cell viability was significantly improved in the Y50 filter, YE, SYD, OO, OG, and GN lens groups as compared with the no-lens group [Figs. 4(a)-4(f)]. Cell viability was not improved in the SY, PN, and reflective coating lens groups as compared with the no-lens group [Figs. 4(g)-4(i)]. 

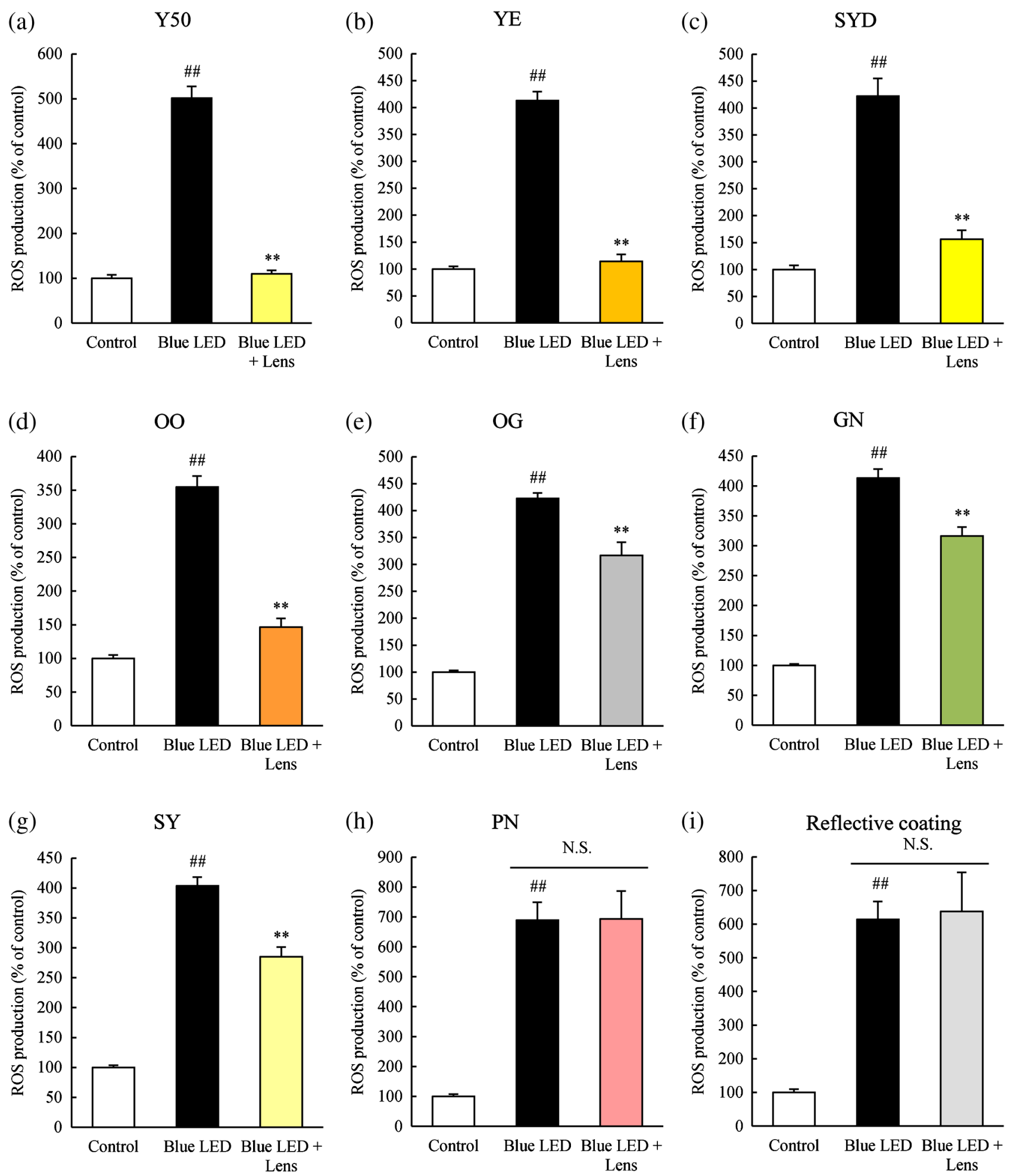

Fig. 5 Effect of colored lenses on ROS production upon blue LED light-induced damage in 661W cells. ROS production was measured by fluorescence intensity $1 \mathrm{~h}$ after the addition of $\mathrm{CM}$ $\mathrm{H}_{2}$ DCFDA, and ROS levels increased upon exposure to blue LED light. (a) Y50 filter, (b) YE, (c) SYD, (d) OO, (e) OG, (f) GN, and (g) SY lenses significantly decreased the level of ROS production compared with the no-lens group. (h) PN and (i) reflective coating lenses did not decrease the level of ROS production compared with the no-lens group. Data are expressed as mean $\pm \operatorname{SEM}(n=6)$. ${ }^{\# \#} p<0.01$ versus control; and ${ }^{* *} p<0.01$ versus blue LED light exposure (one-way ANOVA followed by Tukey's test). 
Table 1 Transmittance of lenses at 400 to $495 \mathrm{~nm}$. The transmittance of lenses at $464 \mathrm{~nm}$ was used for calculating the correlation with their protective effects.

\begin{tabular}{|c|c|c|c|c|c|c|c|c|c|c|c|c|c|c|c|c|c|c|c|}
\hline \multirow{2}{*}{ Wavelength } & \multirow{3}{*}{ Y50 } & \multirow[b]{3}{*}{ YE } & \multirow[b]{3}{*}{ SYD } & \multirow[b]{3}{*}{ OO } & \multirow[b]{3}{*}{$O G$} & \multirow[b]{3}{*}{ GN } & \multirow[b]{3}{*}{ SY } & \multirow[b]{3}{*}{ PN } & \multirow{3}{*}{$\begin{array}{c}\text { Blue light } \\
\text { antireflective } \\
\text { coating }\end{array}$} & \multicolumn{2}{|c|}{$\begin{array}{l}\text { Wavelength } \\
\text { Vens Y50 }\end{array}$} & \multirow{2}{*}{ YE } & \multirow{2}{*}{ SYD } & \multirow{2}{*}{$\mathrm{OO}$} & \multirow{2}{*}{ OG } & \multirow{2}{*}{ GN } & \multirow{2}{*}{ SY } & \multirow{3}{*}{$\frac{\mathrm{PN}}{48.8}$} & \multirow{3}{*}{$\begin{array}{c}\begin{array}{c}\text { Blue light } \\
\text { antireflective } \\
\text { coating }\end{array} \\
80.44\end{array}$} \\
\hline & & & & & & & & & & & & & & & & & & & \\
\hline Vens & & & & & & & & & & $432 \mathrm{~nm}$ & & 9.6 & 4.61 & 10.32 & 27.3 & 252 & 26.7 & & \\
\hline $400 \mathrm{~nm}$ & 0 & 2.7 & 0.7 & 1 & 5.7 & 3.5 & 3.3 & 6.9 & 0.033 & $433 \mathrm{~nm}$ & & 9.7 & 4.81 & 10.92 & 27.4 & 25.62 & 27.5 & 49.5 & 80.929 \\
\hline $401 \mathrm{~nm}$ & & 3.2 & 0.7 & 1.2 & 6.9 & 4.2 & 4 & 8.6 & 0.041 & $434 \mathrm{~nm}$ & & 9.8 & 5.11 & 11.52 & 27.6 & 26.32 & 28.2 & 50 & 81.398 \\
\hline $402 \mathrm{~nm}$ & & 3.7 & 0.8 & 1.3 & 8.1 & 4.9 & 4.7 & 10.3 & 0.048 & $435 \mathrm{~nm}$ & & 10 & 5.41 & 12.12 & 27.7 & 27 & 295 & 50.7 & 81.822 \\
\hline $403 \mathrm{~nm}$ & & 4.1 & 0.9 & 1.4 & 9.3 & 5.7 & 5.4 & 12.2 & 0.039 & $436 \mathrm{~nm}$ & & 10.2 & 5.81 & 12.82 & 27.7 & 27.72 & 29.95 & 51.3 & 82.115 \\
\hline $404 \mathrm{~nm}$ & & 4.5 & 0.9 & 1.6 & 10.5 & 6.4 & 6.2 & 14.2 & 0.022 & $437 \mathrm{~nm}$ & & 10.4 & 6.11 & 13.52 & 27.7 & 28.43 & 30.65 & 51.8 & 82.388 \\
\hline $405 \mathrm{~nm}$ & & 5.1 & 1 & 1.7 & 11.8 & 7.2 & 7 & 16.2 & 0.029 & $438 \mathrm{~nm}$ & & 10.4 & 6.41 & 14.12 & 27.7 & 29.13 & 31.55 & 52.4 & 82.622 \\
\hline $406 \mathrm{~nm}$ & & 5.5 & 1.1 & 1.9 & 12.9 & 7.9 & 7.8 & 18.1 & 0.142 & $439 \mathrm{~nm}$ & & 10.7 & 6.81 & 14.92 & 27.7 & 29.93 & 32.45 & 53.1 & 82.827 \\
\hline $407 \mathrm{~nm}$ & & 5.9 & 1.2 & 2.1 & 14.1 & 8.8 & 8.6 & 20.3 & 0.514 & $440 \mathrm{~nm}$ & 0 & 10.9 & 7.21 & 15.62 & 27.6 & 30.7 & 33.15 & 53.5 & 82.958 \\
\hline $408 \mathrm{~nm}$ & & 6.2 & 1.3 & 2.3 & 15.3 & 9.5 & 9.5 & 22.2 & 1.353 & $441 \mathrm{~nm}$ & & 11.1 & 7.61 & 16.42 & 27.7 & 31.4 & 34 & 54 & 83.153 \\
\hline $409 \mathrm{~nm}$ & & 6.6 & 1.3 & 2.5 & 16.2 & 10.3 & 10.3 & 24.1 & 2.847 & $442 \mathrm{~nm}$ & & 11.3 & 81 & 17.12 & 27.8 & 32.13 & 34.95 & 54.5 & 83.337 \\
\hline $410 \mathrm{~nm}$ & 0 & 6.9 & 1.4 & 2.7 & 17.1 & 11 & 11.1 & 26 & 5.197 & $443 \mathrm{~nm}$ & & 11.4 & 8.5 & 182 & 28.1 & 33 & 35.7 & 55 & 83.49 \\
\hline $411 \mathrm{~nm}$ & & 7.2 & 1.5 & 2.9 & 17.8 & 11.7 & 11.8 & 27.7 & 8.497 & $444 \mathrm{~nm}$ & & 11.7 & 91 & 18.82 & 28.3 & 33.73 & 36.5 & 55.5 & 83.644 \\
\hline $412 \mathrm{~nm}$ & & 7.5 & 1.6 & 3.1 & 18.6 & 12.5 & 12.7 & 29.3 & 12.823 & $445 \mathrm{~nm}$ & & 11.9 & 9.51 & 19.62 & 28.6 & 34.53 & 37.45 & 55.9 & 83.815 \\
\hline $413 \mathrm{~nm}$ & & 7.6 & 1.6 & 3.3 & 19.1 & 13 & 13.3 & 30.9 & 18.017 & $446 \mathrm{~nm}$ & & 12.2 & 102 & 20.52 & 29.3 & 35.43 & 38.45 & 56.4 & 83.925 \\
\hline $414 \mathrm{~nm}$ & & 7.8 & 1.8 & 3.6 & 19.6 & 13.8 & 14 & 32.4 & 23.893 & $447 \mathrm{~nm}$ & & 12.41 & 10.52 & 21.42 & 29.9 & 36.13 & 39.2 & 56.8 & 84.041 \\
\hline $415 \mathrm{~nm}$ & & 8 & 1.8 & 3.8 & 20.1 & 14.4 & 14.8 & 33.8 & 30.182 & $448 \mathrm{~nm}$ & & 12.71 & 11.12 & 22.33 & 30.7 & 37 & 40.15 & 57.3 & 84.14 \\
\hline $416 \mathrm{~nm}$ & & 8.1 & 1.9 & 4 & 20.5 & 15 & 15.5 & 35 & 36.578 & $449 \mathrm{~nm}$ & & 12.91 & 11.72 & 23.33 & 31.7 & 37.8 & 41.15 & 57.7 & 84.205 \\
\hline $417 \mathrm{~nm}$ & & 8.2 & 2 & 4.3 & 20.8 & 15.6 & 16.1 & 36.2 & 42.872 & $450 \mathrm{~nm}$ & 0 & 13.31 & 12.42 & 24.23 & 32.9 & 38.7 & 42 & 58 & 84.234 \\
\hline $418 \mathrm{~nm}$ & & 8.3 & 2.1 & 4.6 & 21.2 & 16.2 & 16.8 & 37.4 & 48.704 & $451 \mathrm{~nm}$ & & 13.7 & 132 & 25.23 & 34.2 & 39.6 & 42.95 & 58.4 & 84.273 \\
\hline $419 \mathrm{~nm}$ & & 8.3 & 2.2 & 4.8 & 21.5 & 16.8 & 17.5 & 38.5 & 53.972 & $452 \mathrm{~nm}$ & & 141 & 13.72 & 26.13 & 35.5 & 40.4 & 43.7 & 58.7 & 84.384 \\
\hline $420 \mathrm{~nm}$ & 0 & 8.4 & 2.3 & 5.2 & 21.9 & 17.4 & 18.2 & 39.6 & 58.616 & $453 \mathrm{~nm}$ & & 14.31 & 14.32 & 27.23 & 36.8 & 41.2 & 44.75 & 59.1 & 84.438 \\
\hline $421 \mathrm{~nm}$ & & 8.6 & 2.4 & 5.4 & 22.3 & 18 & 18.9 & 40.5 & 62.573 & $454 \mathrm{~nm}$ & & 14.51 & 14.92 & 27.93 & 38.2 & 41.9 & 45.45 & 59.3 & 84.456 \\
\hline $422 \mathrm{~nm}$ & & 8.6 & 2.6 & 5.8 & 22.8 & 18.6 & 19.6 & 41.6 & 65.939 & $455 \mathrm{~nm}$ & & 14.81 & 15.52 & 28.93 & 39.6 & 42.8 & 46.35 & 59.6 & 84.579 \\
\hline $423 \mathrm{~nm}$ & & 8.7 & 2.7 & 6.1 & 23.2 & 19.2 & 20.2 & 42.3 & 68.79 & $456 \mathrm{~nm}$ & & 151 & 16.22 & 29.84 & 40.9 & 43.4 & 47.15 & 59.9 & 84.592 \\
\hline $424 \mathrm{~nm}$ & & 8.7 & 2.9 & 6.5 & 23.7 & 19.8 & 20.9 & 43.2 & 71.239 & $457 \mathrm{~nm}$ & & 15.31 & 16.83 & 30.74 & 42.1 & 44.3 & 48 & 60 & 84.655 \\
\hline $425 \mathrm{~nm}$ & & 8.9 & 3 & 6.9 & 24.3 & 20.4 & 21.6 & 44 & 73.276 & $458 \mathrm{~nm}$ & & 15.51 & 17.53 & 31.74 & 43.3 & 45 & 48.8 & 60.4 & 84.656 \\
\hline $426 \mathrm{~nm}$ & & 8.9 & 3.2 & 7.3 & 24.8 & 21 & 22.3 & 44.7 & 74.996 & $459 \mathrm{~nm}$ & & 15.81 & 18.13 & 32.64 & 44.4 & 45.8 & 49.6 & 60.6 & 84.625 \\
\hline $427 \mathrm{~nm}$ & & 9.1 & 3.4 & 7.7 & 25.2 & 21.7 & 23 & 45.4 & 76.427 & $460 \mathrm{~nm}$ & 0 & 16.21 & 18.93 & 33.54 & 45.4 & 46.5 & 50.5 & 60.8 & 84.603 \\
\hline $428 \mathrm{~nm}$ & & 9.1 & 3.6 & 8.2 & 25.7 & 22.2 & 23.7 & 46 & 77.648 & $461 \mathrm{~nm}$ & & 16.51 & 19.53 & 34.34 & 46.2 & 47.2 & 51.1 & 61 & 84.638 \\
\hline $429 \mathrm{~nm}$ & & 9.3 & 3.9 & 8.7 & 26.2 & 23 & 24.5 & 46.7 & 78.512 & $462 \mathrm{~nm}$ & & 16.82 & 20.23 & 35.2 & 47 & 47.8 & 51.9 & 61.1 & 84.668 \\
\hline $430 \mathrm{~nm}$ & 0 & 9.3 & 4 & 9.2 & 26.5 & 23.5 & 25.1 & 47.5 & 79.232 & $463 \mathrm{~nm}$ & & 17.2 & 21 & 364 & 47.7 & 48.5 & 52.6 & 61.1 & 84.671 \\
\hline $431 \mathrm{~nm}$ & & 9.5 & 4.3 & 9.8 & 26.9 & 24.3 & 25.9 & 48.1 & 79.918 & $464 \mathrm{~nm}$ & & 17.52 & 21.83 & 36.74 & 48.2 & 49.2 & 53.3 & 61 & 84.705 \\
\hline
\end{tabular}

Table 1 (Continued). 
Table 1 (Continued)

\begin{tabular}{|c|c|c|c|c|c|c|c|c|c|}
\hline \multicolumn{2}{|c|}{ Wavelength } & \multirow[b]{2}{*}{ YE } & \multirow[b]{2}{*}{ SYD } & \multirow[b]{2}{*}{$\mathrm{OO}$} & \multirow[b]{2}{*}{ OG } & \multirow[b]{2}{*}{ GN } & \multirow[b]{2}{*}{ SY } & \multirow{2}{*}{\multicolumn{2}{|c|}{$\begin{array}{c}\text { Blue light } \\
\text { antireflective } \\
\text { PN } \quad \text { coating }\end{array}$}} \\
\hline Vens & Y50 & & & & & & & & \\
\hline $465 \mathrm{~nm}$ & & 17.9 & 22.5 & 37.5 & 48.8 & 49.7 & 53.9 & 61.1 & 84.626 \\
\hline $466 \mathrm{~nm}$ & & 18.3 & 23.3 & 38.3 & 49.3 & 50.3 & 54.5 & 61.1 & 84.691 \\
\hline $467 \mathrm{~nm}$ & & 18.7 & 24.1 & 38.9 & 49.7 & 50.9 & 55.2 & 61.1 & 84.686 \\
\hline $468 \mathrm{~nm}$ & & 19 & 24.8 & 39.6 & 50 & 51.3 & 55.8 & 61 & 84.671 \\
\hline $469 \mathrm{~nm}$ & & 19.5 & 25.6 & 40.1 & 50.2 & 51.8 & 56.3 & 61 & 84.7 \\
\hline $470 \mathrm{~nm}$ & 0 & 19.9 & 26.4 & 40.6 & 50.6 & 52.4 & 56.9 & 60.7 & 84.729 \\
\hline $471 \mathrm{~nm}$ & & 20.3 & 27.1 & 40.9 & 50.8 & 52.8 & 57.4 & 60.3 & 84.722 \\
\hline $472 \mathrm{~nm}$ & & 20.8 & 27.9 & 41.5 & 51.1 & 53.3 & 57.9 & 60.2 & 84.734 \\
\hline $473 \mathrm{~nm}$ & & 21.3 & 28.7 & 41.9 & 51.3 & 53.7 & 58.6 & 60 & 84.751 \\
\hline $474 \mathrm{~nm}$ & & 21.7 & 29.5 & 42.2 & 51.3 & 54.1 & 58.9 & 59.7 & 84.753 \\
\hline $475 \mathrm{~nm}$ & & 22.2 & 30.2 & 42.5 & 51.6 & 54.7 & 59.5 & 59.4 & 84.723 \\
\hline $476 \mathrm{~nm}$ & & 22.6 & 31.1 & 42.8 & 51.6 & 54.9 & 59.9 & 59.1 & 84.683 \\
\hline $477 \mathrm{~nm}$ & & 23.3 & 31.8 & 43.1 & 51.9 & 55.5 & 60.5 & 58.8 & 84.672 \\
\hline $478 \mathrm{~nm}$ & & 23.6 & 32.5 & 43.2 & 51.9 & 55.8 & 60.8 & 58.4 & 84.732 \\
\hline $479 \mathrm{~nm}$ & & 24.2 & 33.3 & 43.5 & 52.1 & 56.2 & 61.2 & 58.2 & 84.708 \\
\hline $480 \mathrm{~nm}$ & 0.1 & 24.7 & 34 & 43.6 & 52.2 & 56.5 & 61.6 & 57.7 & 84.704 \\
\hline $481 \mathrm{~nm}$ & & 25.3 & 34.7 & 43.8 & 52.3 & 57 & 62.1 & 57.6 & 84.676 \\
\hline $482 \mathrm{~nm}$ & & 25.9 & 35.6 & 44.2 & 52.5 & 57.6 & 62.7 & 57.6 & 84.658 \\
\hline $483 \mathrm{~nm}$ & & 26.6 & 36.5 & 44.6 & 53 & 58.2 & 63.3 & 57.5 & 84.682 \\
\hline $484 \mathrm{~nm}$ & & 27.3 & 37.2 & 45 & 53.2 & 58.6 & 63.7 & 57.6 & 84.737 \\
\hline $485 \mathrm{~nm}$ & & 27.7 & 37.9 & 45.3 & 53.4 & 59 & 64.2 & 57.5 & 84.73 \\
\hline $486 \mathrm{~nm}$ & & 28.4 & 38.6 & 45.5 & 53.6 & 59.5 & 64.6 & 57.4 & 84.696 \\
\hline $487 \mathrm{~nm}$ & & 29.2 & 39.3 & 45.7 & 53.7 & 59.9 & 65 & 57.2 & 84.701 \\
\hline $488 \mathrm{~nm}$ & & 29.7 & 40 & 45.8 & 53.9 & 60.3 & 65.3 & 57.1 & 84.705 \\
\hline $489 \mathrm{~nm}$ & & 30.3 & 40.7 & 46.1 & 53.9 & 60.5 & 65.6 & 56.9 & 84.645 \\
\hline $490 \mathrm{~nm}$ & 8.4 & 31.1 & 41.4 & 46.3 & 54.1 & 60.9 & 66 & 57 & 84.652 \\
\hline $491 \mathrm{~nm}$ & & 31.8 & 42.1 & 46.5 & 54.1 & 61.3 & 66.3 & 56.8 & 84.617 \\
\hline $492 \mathrm{~nm}$ & & 32.5 & 42.7 & 46.6 & 54.3 & 61.5 & 66.6 & 56.6 & 84.611 \\
\hline $493 \mathrm{~nm}$ & & 33.1 & 43.5 & 46.8 & 54.3 & 61.8 & 66.8 & 56.6 & 84.578 \\
\hline $494 \mathrm{~nm}$ & & 33.8 & 44.1 & 46.8 & 54.2 & 62 & 67.1 & 56.4 & 84.541 \\
\hline $495 \mathrm{~nm}$ & & 34.5 & 44.7 & 46.6 & 54.1 & 62 & 67.2 & 56 & 84.525 \\
\hline Average & 0.85 & 15.08 & 815.73 & 323.3 & 334.9 & 235.78 & 338.58 & 348.82 & 268.09827083 \\
\hline
\end{tabular}

\subsection{Reactive Oxygen Species Production Was Decreased in Several Lens Groups}

CM- $\mathrm{H}_{2}$ DCFDA was used as the fluorescent probe for detecting ROS production. The level of ROS production was increased upon exposure to blue LED light. A $350 \%$ to $600 \%$ increase in the level of ROS production was observed in the no-lens group as compared with the control group (Fig. 5). Furthermore, compared with the no-lens group, ROS production was significantly decreased in the Y50 filter, YE, SYD, OO, OG, GN, and SY lens groups [Figs. 5(a)-5(g)]. The level of ROS production in the PN and reflective coating lens groups did not decrease as compared with the no-lens group [Figs. 5(h)-5(i)].

\subsection{Protective Effect of Lenses Correlated with Transmittance}

The transmittance of lenses at 400 to $495 \mathrm{~nm}$ is shown in Table 1. The protective effects of lenses against blue LED light exposure were evaluated by differences in cell viability, level of ROS production, and the rate of cell death in various groups. Here, the protective effects of various lenses were observed to correlate strongly with their transmittance (Fig. 6).

\section{Discussion}

Previous in vivo studies have indicated that yellow lenses protect from retinal damage induced by exposure to white or blue LED light. ${ }^{14-16}$ However, the protective effects of colored lenses have not been investigated in vitro. Recently, we established a blue LED light-induced cell damage model using $661 \mathrm{~W}$ photoreceptor-derived cells. ${ }^{13}$ The current study aimed at investigating the protective effects of colored lenses upon exposure to blue LED light using the previously established model. Further, protective effects of colored lenses and their correlation with lens transmittance at $464 \mathrm{~nm}$ were also evaluated.

ROS levels were found to increase in the no-lens groups, consistent with their reported increase upon blue LED light exposure. ${ }^{13}$ The $661 \mathrm{~W}$ cell was damaged by blue LED light exposure in the no-lens group because ROS-induced oxidative stress and oxidative stress caused photoreceptor cell death and apoptosis. ${ }^{17,18}$ A previous in vivo study has also confirmed photoreceptor cell death and apoptosis induced by blue light exposure. ${ }^{19}$ Our previous report also showed that blue LED light caused S-opsin aggregation related to endoplasmic reticulum (ER) stress. It should be associated with reduction of the oxidative stress and ER stress by colored lenses. Specifically, colored lenses decreased the cell death through the suppression of ROS production in the present study.

The Y50 filter, YE, SYD, OO, OG, and GN lenses displayed highly protective effects against blue LED light exposure, most likely by physically blocking the blue LED light before it arrived at the $661 \mathrm{~W}$ cells and thereby decreasing the blue LED light exposure levels of the $661 \mathrm{~W}$ cells. As a result, $661 \mathrm{~W}$ cell damage was migrated by these lenses. Among these, Y50, the cutting filter below $500 \mathrm{~nm}$, showed the strongest protective effect against $661 \mathrm{~W}$ cell damage induced by blue LED light exposure. Cutting filters lead to changes of transmittance properties in a particular wavelength. The Y50 filter does not transmit below the wavelength of $500 \mathrm{~nm}$. Subsequently, the Y50 filter has the highest blue light absorptive capacity among the various tested colored lenses.

Yellow is known to absorb short wavelengths, consistent with which yellow-colored lenses, namely YE and SYD, also 

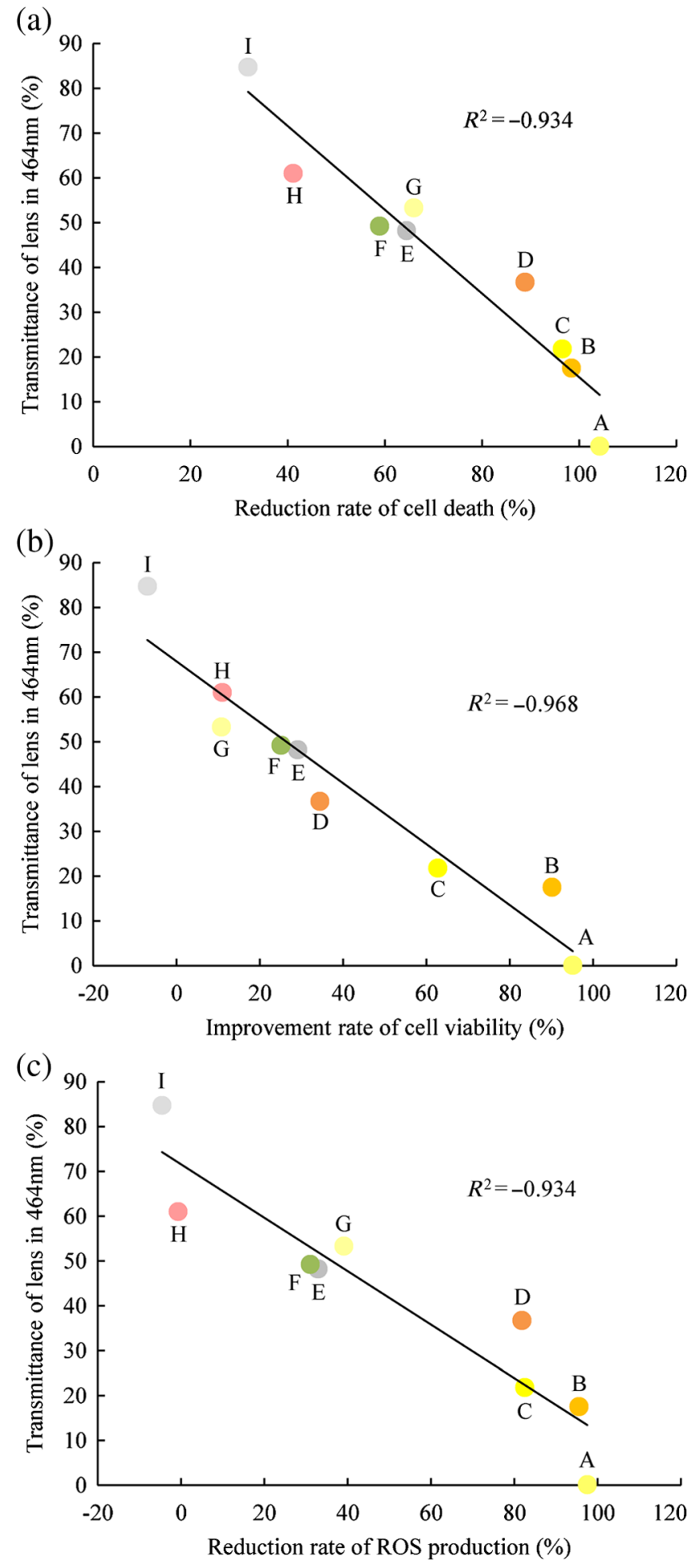

Fig. 6 Correlation of protective effects of lenses with transmittance. The correlations between protective levels of lenses and their transmittance were calculated, and the Pearson product-moment correlation coefficients $\left(R^{2}\right)$ are shown. (a) Decreased rate of cell death; (b) improved cell viability; and (c) decreased ROS production could be correlated with transmittance of lenses.

showed highly protective effects against blue LED light exposure. Recent reports have shown that yellow lenses reduce the expression of blue light-induced inflammatory markers in mice $^{16}$ and have a protective effect against retinal damage induced by blue LED light exposure in rats. ${ }^{14}$ However, clear lenses showed no protective effects against blue light-induced retinal damage in both models. ${ }^{14,16}$ Here, the reflective coating lenses showed no protective effect against $661 \mathrm{~W}$ cell damage owing to their high transmittance at $464 \mathrm{~nm}$, resulting in insufficient blockage and thereby transmittance of blue LED light through these lenses. The lenses with blue light reflective coating showed highest transmittance in the various tested colored lenses and therefore led to cell damage due to direct exposure to blue LED light. Thus, the protective effect of lenses could be correlated with their transmittance at $464 \mathrm{~nm}$. Taken together, these results showed that blue light-induced photoreceptor cell death is correlated with the amount of blue light exposure. Moreover, colored lenses suppressed blue light-induced oxidative stress since the level of ROS production was correlated with lens transmittance at $464 \mathrm{~nm}$.

In conclusion, lenses with low transmittance at $464 \mathrm{~nm}$ protected the $661 \mathrm{~W}$ cells from blue LED light-induced damage. These colored lenses were capable of physically absorbing blue LED light and thereby suppressing blue LED light-induced retinal damage. Our findings also showed that the transmittance of blue light is in large correlation with the protective effect of colored lens in a blue LED light-induced cell damage model. This correlation strongly suggested that an amount of blue light was important in the protection of eyes. Moreover, colored lenses (such as gray and green) except for the yellow lens may have a protective effect on blue LED light-induced retinal damage.

\section{References}

1. M. Hori et al., "Lethal effects of short-wavelength visible light on insects," Sci. Rep. 4, 7383 (2014).

2. M. C. Gordijn, D. 't Mannetje, and Y. Meesters, "The effects of blue-enriched light treatment compared to standard light treatment in seasonal affective disorder," Br. J. Ophthalmol. 136, 72-80 (2012).

3. C. Cajochen et al., "High sensitivity of human melatonin, alertness, thermoregulation, and heart rate to short wavelength light," J. Clin. Endocrinol. Metab. 90(3), 1311-1316 (2005).

4. G. C. Brainard et al., "Action spectrum for melatonin regulation in humans: evidence for a novel circadian photoreceptor," J. Neurosci. 21(16), 6405-6412 (2001).

5. K. E. West et al., "Blue light from light-emitting diodes elicits a dosedependent suppression of melatonin in humans," J. Appl. Physiol. 110(3), 619-626 (2011).

6. C. Roehlecke et al., "Influence of blue light on photoreceptors in a live retinal explant system," Mol. Vision 17, 876-884 (2011).

7. N. Sanvicens et al., "Oxidative stress-induced apoptosis in retinal photoreceptor cells is mediated by calpains and caspases and blocked by the oxygen radical scavenger CR-6," J. Biol. Chem. 279(38), 39268-39278 (2004).

8. J. Cai et al., "Oxidative damage and protection of the RPE," Prog. Retinal Eye Res. 19(2), 205-221 (2000).

9. F. Q. Liang and B. F. Godley, "Oxidative stress-induced mitochondrial DNA damage in human retinal pigment epithelial cells: a possible mechanism for RPE aging and age-related macular degeneration," Exp. Eye Res. 76(4), 397-403 (2003).

10. R. R. Bourne et al., "Causes of vision loss worldwide, 1990-2010: a systematic analysis," Lancet Global Health 1(6), e339-e349 (2013).

11. A. J. Witkin et al., "High-speed ultrahigh resolution optical coherence tomography before and after Ranibizumab for age-related macular degeneration," Ophthalmology 116(5), 956-963 (2009).

12. H. R. Taylor et al., "Visible light and risk of age-related macular degeneration," Trans. Am. Ophthalmol. Soc. 88, 163-178 (1990).

13. Y. Kuse et al., "Damage of photoreceptor-derived cells in culture induced by light emitting diode-derived blue light," Sci. Rep. 4, 5223 (2014). 
14. M. Tanito, S. Kaidzu, and R. E. Anderson, "Protective effects of soft acrylic yellow filter against blue light-induced retinal damage in rats," Exp. Eye Res. 83(6), 1493-1504 (2006).

15. T. Kurihara et al., "Retinal phototoxicity in a novel murine model of intraocular lens implantation," Mol. Vision 15, 2751-2761 (2009).

16. T. Narimatsu et al., "Blue light-induced inflammatory marker expression in the retinal pigment epithelium-choroid of mice and the protective effect of a yellow intraocular lens material in vivo," Exp. Eye Res. 132, 48-51 (2015).

17. R. R. Krishnamoorthy et al., "Photo-oxidative stress down-modulates the activity of nuclear factor- $\kappa \mathrm{B}$ via involvement of caspase-1, leading to apoptosis of photoreceptor cells," J. Biol. Chem. 274(6), 3734-3743 (1999).

18. K. Tsuruma et al., "Role of oxidative stress in retinal photoreceptor cell death in N-methyl-N-nitrosourea-treated mice," J. Pharmacol. Sci. 118(3), 351-362 (2012)

19. J. Wu et al., "Damage of blue light induced apoptosis in rat retina," Eye 13, 577-583 (1999).

Biographies for the authors are not available. 